Pacific Journal of Mathematics 


\title{
MULTIPLICATION OPERATORS
}

\author{
R. C. BuCK
}

1. Introduction. The prototype for partially ordered linear spaces is $C[X]$, the space of all real valued continuous functions on a topological space $X$, with the natural ordering defined by: $f \geqq 0$ if and only if $f(x) \geqq 0$ for all $x \in X$. If $V$ is a real linear space with a partial order defined by a suitable positive cone $P$, then $V$ has a canonical embedding in a function space $C[X]$.

The containing space $C[X]$ has a more elaborate structure than did the original space $V$; in particular, $C[X]$ is an algebra. If we take any aspect of $C[X]$, we may ask how it appears when transferred back to $V$. This paper deals with one aspect of this.

Among the linear operators on $C[X]$, an interesting class that arises in many contexts is the class of multiplication operators. These are defined by:

$$
T(f)=g \quad \text { where } \quad g(x)=\phi(x) f(x) \quad x \in X,
$$

and where $\phi$ is a specific member of $C[X]$.

The central result in this paper is a simple characterization, in terms of order, of the linear operators on $V$ which become multiplication operators when $V$ is represented in a function space $C[X]$. This in turn yields a new and more transparent proof of the Stone-Krein theorem on ordered algebras.

2. A simpler case. Let $V$ be a real linear space. We assume that there is a convex cone $P$ with vertex at 0 which defines an order relation $\leqq$ in $V$ by $x \leqq y$ if and only if $y-x \leqq P$. On $P$, we impose three conditions:

$$
\begin{aligned}
& P \cap-P=\{0\} \\
& P \text { is generating } \\
& P \text { is linearly closed in } V .
\end{aligned}
$$

The second condition implies that every element $x \in V$ is the difference of positive elements; the third condition requires that every line meet $P$ in a (possibly unbounded) closed interval. Note that we do not impose any further lattice properties on $V$, nor do we assume that there is an order unit. If $V^{\prime}$ denotes the dual space of $V$, consisting of all linear functionals on $V$, then $V^{\prime}$ has a natural partial ordering derived from that of $V$. A functional $L$ is said to be positive if $L(x) \geqq 0$ for

Received January 11, 1960. Some of this work was done while the author was a Guggenheim Fellow. 
all $x \geqq 0$; the positive cone in $V^{\prime}$ is $P^{\prime}$. The space $V^{\prime}$ will not in general obey all the properties (1), (2), (3).

Let $\mathscr{L}(V)$ denote the algebra of all linear transformations on $V$. We single out a subclass $\mathfrak{A} \subset \mathscr{L}(V)$ consisting of the order-bounded transformations:

Definition 1. An operator $T \in \mathscr{L}(V)$ is order bounded if there is a constant $r$ such that

$$
-r x \leqq T x \leqq r x \text { for all } x \geqq 0 \text { in } V .
$$

We observe that $\mathfrak{A}$ is a subalgebra of $\mathscr{L}(V)$ containing the identity operator $I$; for, if $T_{1}$ and $T_{2}$ are in $\mathfrak{A}$, with associated constants $r_{1}$ and $r_{2}$, then it follows readily from (4) that $T_{1} T_{2}$ obeys (4) with $r=3 r_{1} r_{2}$. We wish to show that $V$ has function space representations in which the algebra $\mathfrak{A}$ becomes multiplication operators. We will prove this first under the strong restriction that $V$ has an "order unit", and then remove this restriction.

Let us suppose that there is an element $e \in V$ such that $e \geqq 0$ and

$$
\text { for every } x \geqq 0 \text {, there is } \lambda>0 \text { such that } x \leqq \lambda e \text {. }
$$

This restriction can be described geometrically: the point $e$ is a radially interior point of $P$, so that every line thru $e$ meets $P$ in a line segment containing $e$ as interior point.

THEOREM 1. Let $V$ be a partially ordered linear space obeying (1), (2), (3) and (5). Let $\mathfrak{A}$ be the order bounded operators on $V$. Then there is a compact set $\Gamma$ and an order preserving representation $\theta: x \rightarrow \widehat{x}$ of $V$ onto a subspace of $C[\Gamma]$, and an isomorphism $\bar{\theta}: T \rightarrow \hat{T}$ of $\mathfrak{A}$ into the multiplication operators on $C[\Gamma]$ such that

$$
\theta(T x)=\hat{T} \hat{x}
$$

for all $x \in V, T \in \mathfrak{A}$.

Otherwise described, the diagram

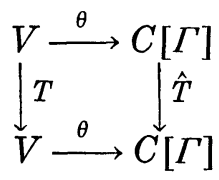

commutes. Corresponding to $T$, there is a function $\phi \in C[\Gamma]$ such that. if $T x=y$, then $\hat{y}(p)=\phi(p) \hat{x}(p)$, for all $p \in \Gamma$.

CoROLlary 1. $\mathfrak{A}$ is a commutative subalgebra of $\mathscr{L}(V)$.

The method we use will be to construct certain appropriate real homomorphisms of $\mathfrak{A}$. Recall first the important notion of a minimal positive element (See Brelot [3] for background.) 
Definition 2. An element $u \geqq 0$ in $V$ is said to be minimal if $0 \leqq x \leqq u$ implies that $x=\lambda u$ for some real $\lambda$.

This can be described geometrically: $u$ is minimal if the ray $\rho$ generated by $u$ is extremal in $P$, and this is so if $u$ cannot be expressed as the midpoint of two points in $P$ that are not on $\rho$. In contrast with the situation for finite dimensional spaces, a cone $P$ in a general linear space will usually have no extremal rays (or minimal elements). This is the case for $C[X]$ when $X$ is the line, but is not the case if $X$ is discrete. The dual cone $P^{\prime}$ of positive linear functionals on $V$ can be better behaved; however, if $V$ is the space $L^{1}[0,1]$, neither $P$ nor $P^{\prime}$ have extremal rays.

Lemma 1. If $P$ is the positive cone in a space $V$ and $P$ contains a radially interior point, then $P^{\prime}$ has a separating family of extremal rays.

This is more or less familiar. (See Bonsall [2], Kadison [8], Kelly [9].) One defines a norm in $V$ by

$$
\|x\|=\inf \{\text { all } r \text { with }-r e \leqq x \leqq r e\} .
$$

Let $D$ be the functionals $L$ on $V$ such that $\|L\| \leqq 1$ and $L(e)=1$. This is then a $w^{*}$ compact convex set in the dual space of $\langle V$, \|\|$\rangle$. Invoking the Krein-Milman theorem, $D$ has extreme points $L_{0}$ whose convex hull is dense in $D$. These are in fact minimal positive elements in $V^{\prime}$, generating extremal rays in $P^{\prime}$. Moreover, if $L_{0}(x)=0$ for all $L_{0}$, then $x=0$.

The key to the proof of Theorem 1 is the observation that minimal elements of $P$ will yield homomorphism of $\mathfrak{A}$ onto the reals. If $T \in \mathfrak{A}$, then by (4) there is a number $r$ such that

$$
0 \leqq r x+T x \leqq 2 r x \quad \text { all } x \geqq 0 .
$$

Let $x=u$, a minimal element of $P$. Then, we see at once that $u$ is an eigenvector for $T$. Denoting the corresponding eigenvalue by $\lambda(T)$, we have $T u=\lambda(T) u$, holding for all $T \in \mathfrak{A}$. But, it then follows that $T \rightarrow \lambda(T)$ is a homomorphism of $\mathfrak{A}$ onto the real field $k$; for, given $T_{1}$ and $T_{2}$, we have

$$
\begin{aligned}
\lambda\left(T_{1} T_{2}\right) u & =T_{1} T_{2}(u) \\
& =T_{1}\left(\lambda\left(T_{2}\right) u\right) \\
& =\lambda\left(T_{1}\right) \lambda\left(T_{2}\right) u .
\end{aligned}
$$

Unfortunately, except in unusual cases, $P$ will not have any minimal elements. Let us go over to the adjoint algebra $\mathfrak{A}^{*} \subset \mathscr{L}\left(V^{\prime}\right)$ consisting of all operators $T^{*}$ for $T \in \mathfrak{A}$. $T^{*}$ is defined on $V^{\prime}$, the dual space of $V$, by: 
and the mapping $T \rightarrow T^{*}$ is an anti-isomorphism of $\mathfrak{A}$ onto $\mathfrak{H}^{*}$. From (7) and (5), we see that if $T$ obeys (4), then

$$
-r L \leqq T^{*}(L) \leqq r L
$$

all $L \geqq 0$.

Thus, $\mathfrak{2}^{*}$ is an algebra of order-bounded operators on the partially ordered space $V^{\prime}$. By Lemma 1 , since $P$ was assumed to have an order unit $e$, there are many minimal elements $L_{0}$ in $P^{\prime}$.

Let $D$ be the convex cross-section of $P^{\prime}$ consisting of all $L \geqq 0$ with $L(e)=1$. Each extreme point of $D$ is a minimal positive element in $P^{\prime}$ and generates an extremal ray; let $\Gamma$ be the closure of the set of extreme points in $D$, in the $w^{*}$ topology arising from the natural norm topology on $V$. By the simple argument given above, each $L_{0} \in \Gamma$ yields a real homomorphism $\lambda_{L_{0}}$ of $\mathfrak{U}^{*}$, defined by the equation

$$
T^{*}\left(L_{0}\right)=\lambda_{L_{0}}\left(T^{*}\right) L_{0} \text {. }
$$

Since $\mathfrak{A}^{*}$ is (anti) isomorphic to $\mathfrak{H}^{*}, \lambda_{L_{0}}$ in turn defines a real homomorphism $h_{L_{0}}$ of $\mathfrak{X}$; using (7), this takes the explicit form:

$$
L_{0}(T x)=h_{L_{0}}(T) L_{0}(x)
$$

all $x \in V$ all $T \in \mathfrak{A}$. all $L_{0} \in \Gamma$

By Lemma 1 , the functionals $L_{0}$ separate $V$ so that the collection of homomorphisms $h_{L_{0}}$ separate $\mathfrak{A}$. We may conclude that $\mathfrak{A}$ is isomorphic to a product of fields $k$, and is therefore commutative; this proves the corollary.

To complete the proof of Theorem 1, we examine (10). We first represent $V$ in $C[\Gamma]$, mapping $x$ onto $\theta(x)=\widehat{x}$ where $\hat{x}\left(L_{0}\right)=L_{0}(x)$ for all $L_{0} \in \Gamma$. Since $L_{0}(e)=1$ for all $L_{0}, \hat{e}$ is the constant function 1 ; in fact, the mapping $\theta$ is one-to-one and order preserving. For fixed $T \in \mathfrak{A}$, define a function $\phi$ on $\Gamma$ by

$$
\phi\left(L_{0}\right)=h_{L_{0}}(T) .
$$

Let $T x=y$; then, (10) can be rewritten as:

$$
\hat{y}\left(L_{0}\right)=\phi\left(L_{0}\right) \hat{x}\left(L_{0}\right) \text {. }
$$

The representation $\theta$ is such that every order-bounded operator $T$ is carried into a multiplication operator on $C[\Gamma]$, and the correspondence is an isomorphism of $\mathfrak{A}$ with a subalgebra of $\mathscr{L}(C[\Gamma])$, and in fact, with a subalgebra of $C[\Gamma]$ itself.

3. The Krein-Stone theorem. Before removing the assumption that $V$ possesses an order unit $e$, we insert an immediate application 
of our results. (See Stone [14], Krein [10], Kadison [8]).

TheOREM 2. Let $A$ be a real algebra with unit $e$ and having a partial order such that if $x \geqq 0, y \geqq 0$, then $x+y \geqq 0$ and $x y \geqq 0$. Assume further that, as a linear space, $A$ obeys restrictions (1), (2), (3) and (5). Then, $A$ is commutative and can be represented as a subalgebra of a function algebra $C[X]$.

Proof. Consider the left regular representation of $A$. This sends $a \in A$ into the operator $U_{a} \in \mathscr{L}(A)$ where $U_{a}(x)=a x$ for all $x \in A$. Since $A$ has a unit, this is an isomorphism of $A$ onto a subalgebra $\bar{A} \subset \mathscr{L}(A)$. By virture of (5), we can choose $r$ depending upon $a$ so that $-r e \leqq a \leqq r e$. If $x \geqq 0$, then $-r x \leqq a x \leqq r x$ so that $U_{a}$ is an order bounded operator on the linear space $\langle A,+\rangle$. Hence, $\bar{A} \subset \mathfrak{A}$, and since this is a commutative algebra, so is $A$.

As a matter of fact, it is not necessary in this proof to assume that $A$ is even associative, since this too can be deduced from the representation. Since $U_{a} U_{b}=U_{b} U_{a}$, it follows that $a(b x)=b(a x)$ for all $x \in A$; with $x=e$, we find that $A$ is commutative. Then, $a(b c)=a(c b)$ while $b(a c)=(a c) b$ and $A$ is associative.

Conversely, we note that Corollary 1 follows from Theorem 2, since $\mathfrak{A}$ itself is an ordered algebra, with $I$ as unit.

Other proofs which have been given for this result rely upon the construction of appropriate real homomorphisms $h$ of $A$. These are linear functionals on $\langle A,+\rangle$ which are multiplicative and obey $h(e)=1$. It is natural to look for these among the extreme points of an appropriate convex set $D$ in the dual space of $\langle A,+\rangle$. Since any finite set of distinct real homomorphisms of $A$ are linearly independent, the collection of $h$ are precisely the extreme points of the convex set $D_{0}$ which they generate. Unfortunately, we cannot obtain $D_{0}$ directly. Instead, one selects a $D \supset D_{0}$, easily described, and then proves $D=D_{0}$. For example, the method adopted in Tate [15], Kadison [8] and Kelley [9] is to select $D$ as all functionals $L$ on $\langle A,+\rangle$ such that $L(e)=1$ and $L\left(x^{2}\right) \geqq 0$ for all $x \in A$. We note that the proof of $D=D_{0}$ depends strongly upon the hypotheses on $A$; one can construct a finite dimensional algebra $B$ for which $D$ is a closed disc, having a circle for its extreme points, but such that $B$ has no proper real homomorphisms.

4. Reduction of the general case. Suppose now that $V$ is not assumed to satisfy (5). This is true for example, of the space $C_{0}[R]$ of functions with compact support, continuous on the real line $R$. We reduce this case to the previous one. Let $e$ be an element in $P$ and form

$$
V(e)=\{\text { all } x \in V \text { such that for some } \lambda,-\lambda e \leqq x \leqq \lambda e\}
$$


This is a linear subspace of $V$; it inherits a partial order from $V$, and in its positive cone $P \cap V(e)$, the element $e$ is an order unit. Suppose that $T \in \mathfrak{A}$. Then, from (4), if $x \in V(e)$, then for the appropriate $\lambda$, we have

$$
-3 \lambda r e \leqq T x \leqq 3 \lambda r e .
$$

Thus, $V(e)$ is left invariant under all operators $T \in \mathfrak{A}$. Accordingly, if we restrict $\mathfrak{A}$ to $V(e)$, we obtain a representation of $\mathfrak{A}$ in $\mathscr{L}(V(e))$. Applying Theorem 1 to the resulting algebra, we find that $\mathfrak{A}$ is commutative in its action on $V(e)$, and also obtain a representation (homomorphic) of $\mathfrak{A}$ as multiplication operators on an appropriate function space $C\left[\Gamma_{e}\right]$. Finally, as $e$ ranges over $P$, the subspaces $V(e)$ cover $V$, and we have proved the following result:

THEOREM 3. Let $V$ be a partially ordered linear space obeying (1), (2) and (3), but not necessarily (5). Let $\mathfrak{A}$ be its algebra of order bounded operators. Then, $\mathfrak{A}$ is commutative, and corresponding to any positive element $e$ in $V$, there is a compact set $\Gamma_{e}$, an order preserving linear representation $\theta$ of $V(e)$ into $C\left[\Gamma_{e}\right]$ and a homomorphism $\bar{\theta}$ of $\mathfrak{A}$ into the multiplication operators on $C\left[\Gamma_{e}\right]$ such that $\theta(T x)=\bar{\theta}(T) \theta(x)$ for all $x \in V(e)$ and $T \in \mathfrak{A}$.

A footnote to this is in order. Although we have shown that the algebra $\mathfrak{A}$ is commutative, we have not shown that it need contain more than the multiples of the identity operator $I$. This can in fact, happen, although it does not in most of the interesting cases discussed in the next section. A glance at the finite dimensional case will be helpful. Let $P$ be a polyhedral cone in $n$-space, and let $u_{1}, u_{2}, \cdots u_{N}$ generate its extremal rays. Each $u_{j}$ is an eigenvector for all the order bounded operators $T \in \mathfrak{A}$, and in turn generates real homomorphisms $h_{j}$ of $\mathfrak{A}$, with

$$
T\left(u_{j}\right)=h_{j}(T) u_{j} .
$$

Suppose that the $\left\{u_{j}\right\}_{1}^{N}$ are such that $N>n$ and every set of $n$ is independent. Then, it follows that all the $h_{j}$ coincide on $\mathfrak{A}$. Since together they define a faithful representation of $\mathfrak{A}$, we conclude that $\mathfrak{A}$ consists exactly of the scalar multiples of $I$. In contrast, if $N=n$, and the $u_{f}$ form a basis, then $\mathfrak{A}$ becomes the algebra of diagonal matrices; these, of course, are the multiplication operators in this representation.

5. Examples. In this section, we give a number of interesting illuastrations of Theorem 3, together with a counterexample to show the necessity of the assumption that $P$ is a linearly closed cone.

First, choose $V$ as the space $C_{0}[X]$ of all real valued continuous functions on the locally compact space $X$ which vanish at infinity. With 
the usual ordering ( $f \geqq 0$ means $f(p) \geqq 0$ for all $p \in X$ ) this is a partially ordered linear space satisfying the hypotheses of Theorem 3. Note in particular that $C_{0}[X]$ does not have an order unit. What are the order bounded operators on $C_{0}[X]$ ? Applying Theorem 3, we choose any $e \geqq 0$ in $C_{\mathrm{c}}[X]$ and form the subspace $V(e)$. By (13), $f \in V(e)$ if and only if $f / e$ is a bounded function on $X$. Thus, $V(e)$ is isomorphic to the space of bounded continuous functions on the open support $O_{e}$ of $e$. The set $\Gamma_{e}$ is the Cech compactification of $O_{e}$, which contains $O_{e}$ densely. Any point $p \in O_{e}$ defines a minimal functional $L_{p}$ on $V(e)$ so that by (10) and (12),

$$
L_{p}(T f)=(T f)(p)=\phi(p) f(p)
$$

for all $p \in 0_{e}$ and any $T \in \mathfrak{A}$. If $X$ is $\sigma$-compact, we can take $e$ so that $O_{e}=X$, and we find that the only order bounded transformations on $C_{0}[X]$ are those defined as point-wise multiplication by bounded continuous functions $\phi$ on $X$. If $X$ is not $\sigma$-compact, we arrive at the same conclusion by varying $e$.

We note that if $V$ is $C[X]$ itself, a simple and direct characterization of the order bounded operators is available. Using the fact that if $f\left(p_{0}\right)=0$, then we may write $f=f_{1}-f_{2}$ where $f_{i} \geqq 0$ and $f_{i}\left(p_{0}\right)=0$, it readily follows from the characteristic property of $T$ that $(T f)\left(p_{0}\right)=0$. Applying this to $f=g-g\left(p_{0}\right)$, we have $T g=\phi g$ where $\phi=T(1)$.

Another interesting special case is obtained by taking $V$ as the space $H$ of all bounded harmonic functions on an open domain $\Omega$. The constant function is an order unit for $H$ so that we do not need the full machinery of Theorem 3. The extremal rays in $P$ are generated by the R. S. Martin minimal functions (see Brelot [3]) and $H$ is represented as a subspace of the space of continuous functions on the ideal boundary $\Gamma$ of $\Omega$. The order bounded transformations are represented in turn as $C[\Gamma]$ itself; for any $T \in \mathfrak{A}, T f$ is the harmonic function $g \in H$ which is described by the (abstract) Dirichlet problem $\left.g\right|_{\Gamma}=\left.\phi f\right|_{r}$ where $\phi$ is the function in $C[\Gamma]$ corresponding to $T$. Note that $T$ is not a multiplication on $\Omega$ itself. With $\Omega$ chosen as the unit disc and $\phi(x, y)=x$, we have $T(1)=x, T(y)=x y$, but $T(x)=(1 / 2)\left\{x^{2}-y^{2}+1\right\}$, and $T(x y)=$ $(1 / 4)\left\{3 x^{2} y-y^{3}+y\right\}$.

A somewhat more complicated illustration is provided by the space $C[X: E]$ of all bounded functions $f$ on a locally compact space $X$ with values in a fixed partially ordered linear space $E$. We order this by saying $f \geqq g$ when $f(p) \geqq g(p)$ for all $p \in X$. We shall also assume that $E$ has an order unit $e$ and require that each $f$ be continuous when $E$ is given the norm topology associated with $e$. If $v \in E$, denote by $\bar{v}$ the constant function on $X$ with value $v$. Note that $\bar{e}$ is then an order unit for $C[X: E]$. To apply Theorem 3 , we must determine minimal functionals in the dual space of $V$. We can find one associated with each point 
$p_{0} \in X$ and any minimal functional $\theta$ on $E$; define $L_{0}$ on $C[X: E]$ by $L_{0}(f)=\theta\left(f\left(p_{0}\right)\right)$. The following argument proves that $L_{0}$ is indeed minimal. Suppose $0 \leqq L \leqq L_{0}$. Then, for any $v \geqq 0$ in $E, 0 \leqq L(\bar{v})=$ $\theta(v)$. Thus, $v \rightarrow L(\bar{v})$ is a positive linear functional on $E$ which is dominated by $\theta$. Since $\theta$ is minimal on $E$, there is a constant $\rho$ such that $L(\bar{v})=\rho \theta(\bar{v})=\rho L_{0}(\bar{v})$ for all $v \geqq 0$ in $E$ (and thus for all $v \in E$ ). Suppose now that $f \in C[X: E]$ with $f(p) \leqq f\left(p_{0}\right)$ for all $p \in X$; we shall say that such a function $f$ takes a maximum value at $p_{0}$ and that $f \in \mathscr{F}_{p_{0}}$. Setting $v=f\left(p_{0}\right)$, we have $\bar{v}-f \geqq 0$ so that $0 \leqq L(\bar{v}-f) \leqq L_{0}(\bar{v}-f)$. But, $L_{0}(\bar{v}-f)=\theta\left(v-f\left(p_{0}\right)\right)=0$ so that $L(f)=L(\bar{v})=\rho L_{0}(\bar{v})=\rho L_{0}(f)$. Thus, $L=\rho L_{0}$ on the linear span of the special class $\mathscr{F}_{p_{0}}$. Consider now a general function $F \in C[X: E]$; since $F$ is bounded, $\|F(p)\| \leqq M$ for all $p \cong X$. Define $g, g_{1}$, and $g_{2}$ on $X$ by:

$$
\begin{aligned}
g(p) & =F(p)-F\left(p_{0}\right) \\
g_{1}(p) & =\frac{1}{2}\{2\|g(p)\| e+g(p)\} \\
g_{2}(p) & \left.=\frac{1}{2}\{2\|g(p)\| e-g(p)\}\right\}
\end{aligned} \quad p \in X .
$$

One sees that $g_{i} \geqq 0$ and $g_{i}\left(p_{0}\right)=0$, with $\left\|g_{i}(p)\right\| \leqq 3 M$ for all $p \in X$. Moreover,

$$
g(p)=\left\{4 M-g_{2}(p)\right\}-\left\{4 M-g_{1}(p)\right\}
$$

for all $p \in X$, so that $g \in \mathscr{F}_{p_{0}}-\mathscr{F}_{p_{0}}$. We conclude that $L(F)=\rho L_{0}(F)$, so that $L_{0}$ is indeed a minimal positive functional on $C[X: E]$.

Let $\Gamma$ be the set of extreme points in the set $D$ of functionals $\alpha$ on $E$ with $\alpha \geqq 0$ and $\alpha(e)=1$. Applying Theorem 3, we find that any order bounded operator $T$ has the property that

$$
\alpha\left(T(f)\left(p_{0}\right)\right)=\alpha\left(T(\bar{e})\left(p_{0}\right)\right) \alpha\left(f\left(p_{0}\right)\right)
$$

for all $f \in C[X: E], p_{0} \in X$ and $\alpha \in \Gamma$. If we represent the functions $f$ in $C[X: E]$ as functions $f$ on $X \times \Gamma$, then

$$
\bar{\theta}(T f)(p, \alpha)=\phi(p, \alpha) f(p, \alpha)
$$

for all $(p, \alpha)$.

The original space $C[X: E]$ is not an algebra, but is a module over the algebra $C[X]$. Formula (9) shows immediately that any order bounded transformation on $C[X: E]$ is in fact algebraic. If $\psi \in C[X]$ and $f \in C[X: E]$, then $T(\psi f)=\psi T(f)$. For,

$$
\begin{aligned}
\alpha(T(\psi f)(p)) & =\phi(p, \alpha) \alpha(\psi(p) f(p)) \\
& =\psi(p) \phi(p, \alpha) \alpha(f(p)) \\
& =\psi(p) \alpha(T(f)(p)) \\
& =\alpha(\psi(p) T(f)(p))
\end{aligned}
$$


for each $p \bumpeq X$ and $\alpha \in \Gamma$.

Finally, we use a familiar example to show that the most crucial hypothesis on the partially ordered linear space $V$ in Theorem 1 and 3 is that $P$ be linearly closed. Take for $V$ the space of all polynomials, with the ordering: $a_{0}+a_{1} x+\cdots+a_{m} x^{m}>0$ if $a_{m}>0 . \quad P$ satisfies the first and second requirements, but is not linearly closed; in fact

$$
\lambda\left(x^{2}\right)+(1-\lambda)(-x) \in P \text { only if } \lambda>0 .
$$

There is no order unit. We can still introduce the algebra $\mathfrak{A}$ of order bounded transformations on $V$. It is easy to see, however, that $\mathfrak{A}$ is not commutative. Let $T$ be defined on $V$ by $T\left(x^{n}\right)=q_{n}$ where $q_{n}$ is a polynominal of degree less than $n$. Then, $I \pm T \geqq 0$ so that $T \in \mathfrak{A}$. In particular, $T_{1}=x\left(d^{2} / d x^{2}\right)$ and $T_{2}=d / d x$ are in $\mathfrak{A}$; however, $T_{1} T_{2} \neq T_{2} T_{1}$. In this example, the reason for this can be traced to the fact that $P$ is so large that there are too many positive linear operators on $V$, (and no non-degenerate positive linear functionals).

\section{REFERENCES}

1. G. Birkhoff, Lattice Theory, Amer. Math. Soc. Colloq. No. 25 (Rev. edition) 1948.

2. F.F. Bonsall, Sublinear functionals and ideals in partially ordered vector spaces, Proc. London Math. Soc. 4 (1954), 402-418.

3. M. Brelot, Topology of R.S. Martin and Green lines, Lectures on Functions of a complex variable, University of Michigan Press, (1955), 105-121.

4. R. C. Buck, A factoring theorem for homomorphisms, Proc. Amer. Math. Soc., 2 (1951), 135-137.

5. H. Cartan, Un théorème sur les groupes ordonnés, Bull. Sci. Math., 63 (1939), 201-205.

6. P. C. Curtis, Order and commutativity in Banach algebras, Proc. Amer. Math. Soc., 9 (1958), 643-645.

7. N. Jacobson, Structure theory for algebraic algebras of bounded degree, Annals of Math., 46 (1945), 695-707.

8. R. V. Kadison, A representation theory for commutative topological algebras, Memoirs Amer. Math. Soc. No. 7, (1951).

9. J. L. Kelley and R. L. Vaught, The positive cone in Banach algebras, Trans. Amer. Math. Soc., 74 (1953), 44-55.

10. S. Krein, I. Vernikov and A. Tovbin, Sur les anneaux semiordonnés, C. R. (Doklady) Akad. Soc. USSR, 30 (1941), 785-787.

11. N. McCoy, Rings and ideals, Carus Monograph No. 8, (1948).

12. N. McCoy and A. Forsythe, On the commutativity of certain rings, Bull. Amer. Math. Soc., 52 (1946), 523-526.

13. S. Sherman, Order in operator algebras, Amer. J. Math., 73 (1951), 227-232.

14. M. H. Stone, A general theory of spectra, I, Proc. Nat. Acad. Sci., 26 (1940), 280-283.

15. J. T. Tate, On the relation between extremal points of convex sets and homomorphisms of algebras, Commun. Pure and Applied Math., 4 (1951), 31-32.

16. F. B. Wright, Hölder groups, Duke Math. J., 24 (1957), 567-571. 



\section{PACIFIC JOURNAL OF MATHEMATICS}

\section{EDITORS}

Ralph S. Phillips

Stanford University

Stanford, California

F. H. BrownelL

University of Washington

Seattle 5 , Washington
A. L. Whiteman

University of Southern California Los Angeles 7. California

L. J. PAIGE

University of California

Los Angeles 24, California

ASSOCIATE EDITORS
E. F. BECKENBACH
D. DERRY
H. L. ROYDEN
E. G. STRAUS
T. M. CHERRY
M. OHTSUKA
E. SPANIER
F. WOLF

\section{SUPPORTING INSTITUTIONS}

UNIVERSITY OF BRITISH COLUMBIA

CALIFORNIA INSTITUTE OF TECHNOLOGY

UNIVERSITY OF CALIFORNIA

MONTANA STATE UNIVERSITY

UNIVERSITY OF NEVADA

NEW MEXICO STATE UNIVERSITY

OREGON STATE COLLEGE

UNIVERSITY OF OREGON

OSAKA UNIVERSITY

UNIVERSITY OF SOUTHERN CALIFORNIA
STANFORD UNIVERSITY

UNIVERSITY OF TOKYO

UNIVERSITY OF UTAH

WASHINGTON STATE COLLEGE,

UNIVERSITY OF WASHINGTON

AMERICAN MATHEMATICAL SOCIETY

CALIFORNIA RESEARCH CORPORATION

HUGHES AIRCRAFT COMPANY

SPACE TECHNOLOGY LABORATORIES

NAVAL ORDNANCE TEST STATION

Printed in Japan by International Academic Printing Co., Ltd., Tokyo, Japan

Reprinted 1966 in the United States of America 


\section{Pacific Journal of Mathematics}

\section{Vol. 11, No. $1 \quad$ November, 1961}

A. A. Albert, Generalized twisted fields ............................ 1

Richard Arens, Operational calculus of linear relations ................... 9

John Herbert Barrett, Disconjugacy of a self-adjoint differential equation of the fourth order ....................................... 25

Paul Richard Beesack, Hardy's inequality and its extensions ............... 39

Julius Rubin Blum and David Lee Hanson, On invariant probability measures.

II .............................................

Robert Allen Bonic, Symmetry in group algebras of discrete groups.......... 73

R. Creighton Buck, Multiplication operators ...................... 95

Jack Gary Ceder, Some generalizations of metric spaces ................. 105

Meyer Dwass, Random crossings of cumulative distribution functions ......... 127

Albert Edrei, Wolfgang H. J. Fuchs and Simon Hellerstein, Radial distribution and

deficiencies of the values of a meromorphic function ............... 135

William Cassidy Fox, Harmonic functions with arbitrary local singularities ..... 153

Theodore Thomas Frankel, Manifolds with positive curvature ............... 165

Avner Friedman, A strong maximum principle for weakly subparabolic

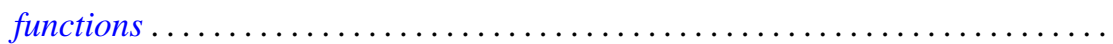

Watson Bryan Fulks and J. O. Sather, Asymptotics. II. Laplace's method for

multiple integrals ......................................

Adriano Mario Garsia and Eugene Richard Rodemich, An embedding of Riemann

surfaces of genus one ..................................... 193

Irving Leonard Glicksberg, Weak compactness and separate continuity......... 205

Branko Grünbaum, On a conjecture of H. Hadwiger .................. 215

Frank J. Hahn, On the action of a locally compact group on $E_{n} \ldots \ldots \ldots \ldots \ldots . . \ldots 221$

Magnus R. Hestenes, Relative hermitian matrices ..................... 225

G. K. Kalisch, On similarity invariants of certain operators in $L_{p} \ldots \ldots \ldots \ldots .247$

Yitzhak Katznelson and Walter Rudin, The Stone-Weierstrass property in Banach

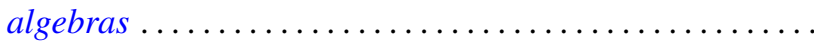

Samir A. Khabbaz, The subgroups of a divisible group $G$ which can be represented as intersections of divisible subgroups of $G \ldots \ldots \ldots \ldots \ldots \ldots \ldots \ldots \ldots . \ldots \ldots 7$

Marvin Isadore Knopp, Construction of a class of modular functions and forms .......................................... 275

Charles Alan McCarthy, Commuting Boolean algebras of projections .......... 295

T. M. MacRobert, Transformations of series of E-functions ................ 309

Heinz Renggli, An inequality for logarithmic capacities ................. 313

M. S. Robertson, Applications of the subordination principle to univalent functions .......................................... 315

David Sachs, Partition and modulated lattices ..................... 325

Frank S. Scalora, Abstract martingale convergence theorems ............... 347

Elbert A. Walker, Torsion endomorphic images of mixed Abelian groups ........ 375

Morgan Ward, The prime divisors of Fibonacci numbers................. 379

Charles R. B. Wright, On the nilpotency class of a group of exponent four....... 387 